\title{
Epidemiology of Thoracolumbar Spine Injuries Associated with Speedboat Travelling without Collision: A Unique Spine Injury Mechanism
}

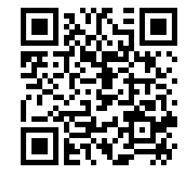

\author{
Narote Treenarong* \\ Department of Orthopedic, Bangkok Hospital Phuket, Thailand
}

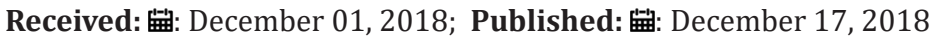

*Corresponding author: Narote Treenarong, Department of Orthopedic Bangkok Hospital Phuket 2/1 Hongyok-Utis Road, Phuket 83000, Thailand

Abstract

Objective: To evaluate the epidemiology of thoracolumbar spine injuries following speedboat travelling at our coastal tertiary trauma center, and to identify injury patterns and mechanism of injury of these specific injuries and to determine the association of position on board with the risk of thoracolumbar injury in this type of transportation.

Materials and Methods: A retrospective review of a consecutive series of 80 patients sustaining thoracolumbar spine injuries following speed boating was conducted. The enrolled patients were treated at Level II trauma center over a 2-year period since July 1, 2016 to May 31, 2018. All the victims were unrestrained passengers on board a speedboat travelling at high speed without any safety regulation. Initially full evaluation with advance trauma life support protocol on arrival was performed. Plain radiographs, computed thermography or MRI study was obtained individually. Number, level and type of thoracolumbar injury were recorded and reported base on CT scan and Thoracolumbar Injury Classification by AO group. The association of the position on board with incidence of thoracolumbar spine injury was then analysed and compared relatively.

Results: All spinal fractures are single level injury. The level of injury is mainly at thoracolumbar area that involved 6 cases (7.5\%) of T11 vertebra, 20 cases (25\%) of T12 vertebra, 44 cases (55\%) of L1 vertebra and 10 cases (12.5\%) of L2 vertebra respectively. No injury of cervical and sacral region has been reported. A spinal cord/nerve root injury occurred in the lumbar region in 1 patient (1.25\%). All of 80 injuries patterns are type A injury (compression type), classified by AO Spine Thoracolumbar Injury Classification, including of 32 levels ( $40 \%$ ) of subtype A1, 18 levels (22.5\%) of subtype A2, 8 levels (10\%) of subtype A3 and 22 levels (27.5\%) of subtype A4. Patients who sat in the front row seat were significantly at higher risk for thoracolumbar spine injury ( $\mathrm{p}<0.05$; odds ratio $=41.83 ; 95 \% \mathrm{CI}: 15.24,114.8$ ). No patient who sat at the rear was reported having this kind of injury.

Conclusion: Our data show the high incidence of this unique compressive type of thoracolumbar spine injury in the patient who sat in the front row seat while on boarding speed boat traveling. As a result of increasing recognition of these regional-specific injuries, practitioners who take responsibility for trauma patient on the coastal area should be aware of the high prevalence of these injuries. Proper universal spinal precaution must be followed to optimize treatment outcome. Current safety regulations including of speed limitation, proper safety restrain technique, bracing position on board and marine rescuers should be reviewed or changed to minimize the number of the victim and degree of damage.

Keywords: Thoracolumbar Spine; Spine Injury; Speedboat; Epidemiology

Abbreviations: CT: Computed Tomography; MRI: Magnetic Resonance Imaging; T12: Thoracic Region

\section{Introduction}

Several studies have been reported on spinal fracture epidemiology in specific high-energy mechanisms setting [1-3], such as motor vehicle collision, fall from height and contact sporting activities. However, it is not only the terrestrial accident that can be the cause of spinal injury, but also the coastal accident too. On the coastal region, there are a variety of water-based recreational activities that predispose to spinal and other traumatic injury [4]. These include personal motorized watercraft (AKA; Jet ski, Waverunner), speed boating, surfing, kayaking and body surfing. As increasing in its popularity, more recently, the number of accident 
report has been documented extensively in the literature. Many studies reported spinal injuries following these water-related recreational activities [5-9]. However, only a small number of studies documents of uncommon spine injury following speedboat travelling without collision and its actual mechanism of injury [10]. If the personal watercrafts are the motorcycles of the water, then the speedboats are certainly the cars. These aquatic vehicles travel at high speed across the water and their passengers are rarely wearing any protective equipment. Unfortunately, these activities are associated with significant risk of spinal injury when accidents occur. Annually, there is an increase in tourism to these regions and many of the tourists are less familiar with the risks and dangers associated with these aquatic vehicles. This is the reason why during high season period, the coastal city is often accompanied by a surge of traumatic incidents associated with the aquatic recreational activities [11,12].

It is commonly known that spinal injuries are most common resulting from several high-energy mechanisms [13-19] include motor vehicle accident, fall from height [20], contact sporting activities and extreme sport activities [16-18]. Among these spinal injuries, thoracolumbar spine area accounted for a majority of all spinal injuries [21-23]. Anatomically, this area is a transitional zone where the less mobile and rigid thoracic cages change to the more mobile lumbar spine. Thoracolumbar spine injuries are devastating injuries that can result in permanent impairment of function, when accompanying with spinal cord/nerve injury, and loss of quality of life. In some circumstance, these can be lifethreatening condition [24]. To obtain the optimum outcome of treatment, the health care teams should be well trained in caring for these injured patients with current spinal precaution protocol and treatments. By examining the epidemiology of injuries arising from this regional-specific setting, it is believed that more favourable treatment outcomes can be obtained through efficient safety regulation and injury management either non-operative or operative management. The purposes of our study were to evaluate the epidemiology of thoracolumbar spine injuries associated with speedboat travelling without collision at our coastal tertiary trauma center, and to identify its injury patterns and mechanism of injuries and to determine the association of position on board with the risk of thoracolumbar injury in this type of transportation. In addition, we discuss the current safety regulation and encourage the need to improve the law regarding marine transportation act.

\section{Materials and Methods}

Institutional review board approval was obtained for this study. All patients were retrospectively identified from a prospectively collected database of patients admitted or treated at the emergency department, out-patient orthopaedic clinic and in-patient service of the authors' local tertiary trauma center which situated on Phuket, the largest island on the southern part of Thailand. Patients enrolled from July 1, 2016 to May 31, 2018. There were 4,882 trauma patients treated or admitted during this time frame. Eligible patients were identified from ICD-10 data in the context of thoracic or lumbar spine fracture. This led to 118 patients eligible for inclusion. Traumatized patients who did not meet the inclusion criteria of having a mechanism of thoracolumbar spinal injury following speed boating were then excluded ( $N=38$ ). Finally, total 80 patients were left for our study. All the victims were unrestrained passengers on board a speedboat travelling at high speed without any safety regulation. They were fully evaluated with advance trauma life support and spinal precaution protocol initially. All of the patients sustaining thoracolumbar spine injuries were obtained lateral radiographs of cervical spine, both AP and lateral radiographs of thoracolumbar spine and CT scan of whole spine to identify the morphology of spinal fracture. The MRI studies were obtained when presenting of neurological deterioration or necessity of evaluation of posterior ligamentous complex integrity (Figures $1 \& 2$ ).

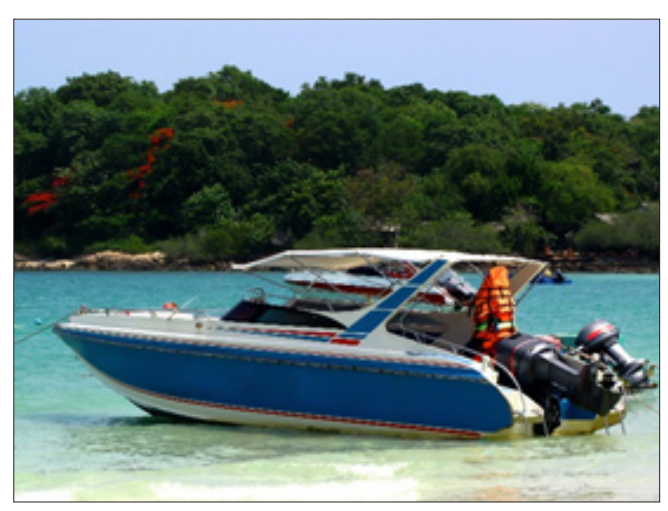

Figure 1: Speedboat (Open motor boating). The most popular transportation in coastal area.

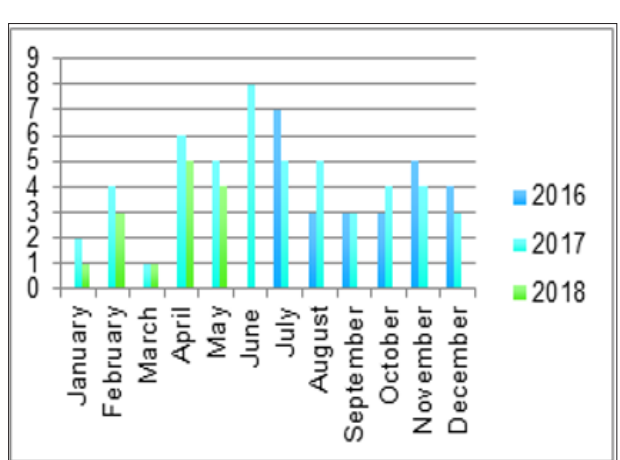

Figure 2: Monthly incidence report of thoracolumbar injury following speed boating.

\section{Results}

All spinal fractures occurring in our patients are single level injury with a total number of 80 spinal levels from 80 victims sustaining thoracolumbar spine injuries following speed boating. The level of injury is located mainly at thoracolumbar area that involved 6 cases $(7.5 \%)$ of T11 vertebra, 20 cases $(25 \%)$ of T12 vertebra, 44 cases $(55 \%)$ of L1 vertebra and 10 cases $(12.5 \%)$ of L2 vertebra. No injury of cervical and sacral region has been reported. The spinal cord/nerve root injury occurred at the thoracic region (T12) in only 1 patient (1.25\%) whom presented with incomplete paraplegia (Figures $3 \& 4$ ). All of 80 victims were unrestrained on boarding. Thoracolumbar spine injuries were reported on 76 victims (3.38\%) who sat at the front row seat and 4 patients 
$(0.18 \%)$ who were at the middle. No thoracolumbar spinal injury has been reported in our patients who sat at the rear of the boat. Patients who sat in the front row seat were significantly at higher risk for thoracolumbar spine injury ( $\mathrm{p}<0.05$; odds ratio $=41.83$; 95\%CI: 15.24, 114.8) (Figure 5).

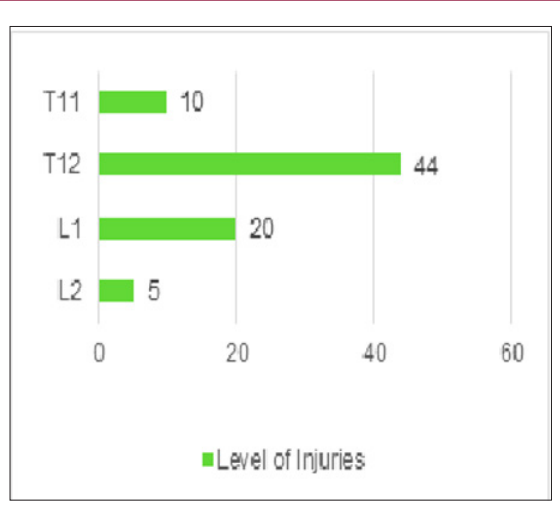

Figure 3: The most common affected vertebra following speed boating accident is T12 vertebra followed by L1 vertebra.

\section{Type A injury (Compression Type)}

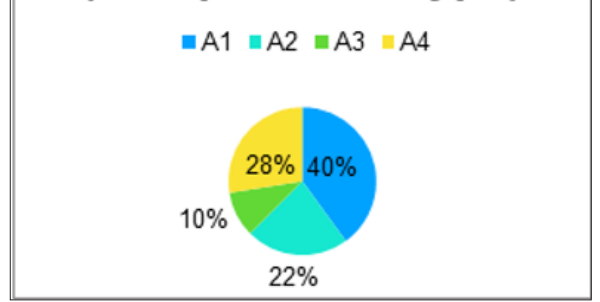

Figure 4: Classified by AO Spine TL Injury Classification, all fracture patterns in our study are type A (compression type) and divided in subtypes. A1: Compression; A2: Pincer; A3: Incomplete burst.
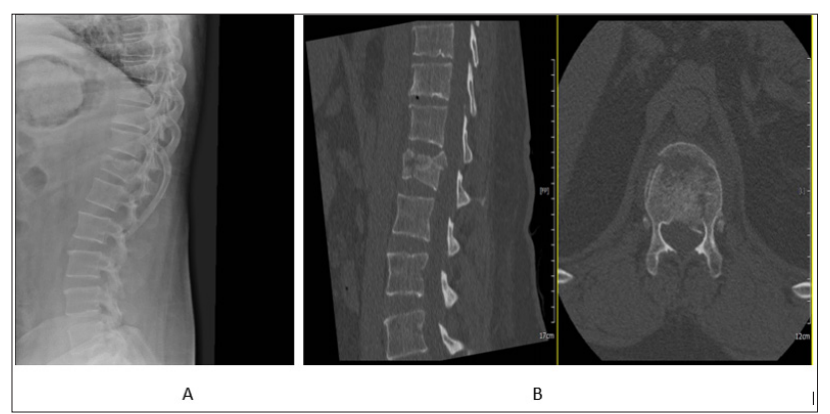

Figure 5: Imaging study of thoracolumbar spine injury following speed boating.

A: Lateral radiographs of TL spine showed anterior wedge compression fracture of T12 vertebra.

B: CT scan revealed disruption of posterior vertebral body with retropulsion which is indicated.

\section{Discussion}

These data indicate that the spinal injuries following speedboat travelling without collision are located mainly at thoracolumbar junction. This transitional zone of the thoracolumbar junction accounts for the majority of thoracic and lumbar spine fractures with $90 \%$ of these injuries occurring between T11and L4 vertebrae $[22,23,25]$. On the terrestrial injuries, thoracolumbar spinal fractures are frequently common following a fall and high velocity automotive accidents with a higher incidence among unrestrained occupants [26-29]. In coastal injuries, however, the incidence of thoracolumbar spinal fractures is less common and have only been reported following diving [30], and water-skiing accidents [5,6]. Speedboat accidents, especially without collision, are uncommon. Previous literature review did not reveal any documented cases of thoracolumbar spinal injuries as a result of speed boating without collision. To our knowledge, this is the first large-scale reported case series of their kind. The fracture morphology presented in our study is only type A (compression type) classified by $\mathrm{AO}$ classification. Although, compression injuries are the most common injury to the thoracolumbar spine, representing $48 \%$ of all thoracolumbar spine fractures and $58 \%$ of all major spinal injuries [31,32], tension band and displacement/translation injuries had been observed in several high-energy mechanism of injury as well. It is very interesting that there was no any tension band injury or displacement/translation injury observed in our study.

The fracture morphology confirmed by CT scan represent only compression type of fracture which were indicated that compression injury is a unique characteristic of thoracolumbar fracture following speed boating. A fracture classification, based on the three-column theory reported by Denis [31,32] and more recently by the AO group [33] is essential for the further understanding of stability of thoracolumbar spine fractures. The unstable spine is one in which progressive anatomic deformity or neurological deficit will occur under continued stress [31,32]. By definition, any fracture involving the middle column (posterior portion of the vertebral body, posterior longitudinal ligament, and the posterior annulus fibrosus) is inherently unstable [31]. These features were observed in one-third of our patients, who all had A-type fractures [33]. These compression injuries, as observed in our case series, represent a failure under compressive forces on the anterior column and/or the middle column. In two-third of our patients who had subtype A1, A2 injuries the middle column remained intact acting as a hinge. The posterior vertebral body was maintained and there was no subluxation. While the other one-third had subtype A3, A4 injuries involving posterior vertebral body disruption representing failure of middle column which is considered unstable spinal injury. Thoracolumbar spinal fractures following road traffic accidents are mostly flexion injuries [34].

Thoracic spine injury in the unrestrained motorcyclist is thought to occur as the result of hyper-flexion of the spine on impact with objects [35], with maximal loading and injury at the level of the mid thoracic vertebrae (T4-T7) $[27,36]$. The higher incidence of low levels of thoracic spinal injury in car occupants may reflect protection of the thoracolumbar spine afforded by the seat and seat belt [37]. In our series, the victims report the mechanism of the injury involved an event including flexion of their spine during the boat's climbing on to the wave followed by sudden deceleration of the boat when landed back on to the water surface 
that resulted in sudden flexion and axial compression loading of their thoracolumbar spine. This mechanism was observed in all of the reported cases, resulting in compression type injuries, based on the Allen-Ferguson classification [38], at different levels of the thoracolumbar spine of the unrestrained speedboat victims. More importantly, our case series show the association of the position of the seat when on board with the risk of thoracolumbar spine injury. The patients who sat at the front are at highest risk for sustaining thoracolumbar spine injury. This could be imagining by the speedboats contain their engine on the rear, resulting in elevation of the front part of the boat, which is at least 1-1.5 meter higher than the water surface.

When the boat encountered the big wave at their speed, the boat was suddenly halted, climbing up on to the wave and landed back forcefully on the water surface. This is resembled to actual mechanism of fall from height that caused a sudden forward flexion and axial compressive load to the thoracolumbar spine area. Furthermore, the center of gravity of human body is in front of thoracolumbar area causing the anterior and middle column of thoracolumbar spine are always under compressive force while the posterior column is under tensile force. All of these support the unique injury findings that all thoracolumbar fractures occurred in our case series are compressive type. A limitation of this study was the retrospective nature of the analysis that may be more susceptible to selection bias. Although all data were collected prospectively, it was not recorded with the current study in mind and it is possible that some pertinent data may have been overlook. In fact, each boat occupancy rate might slightly differ from what we calculated by using 100\% occupancy rate and might result in change of statistical significance of disease/injury association.

\section{Conclusion}

We found high incidence of thoracolumbar spine injury associated with speed boating without collision. Most injuries were compression fracture between Th11-L4 level. The majority of cases reported sitting at the front row seats of speed boats. As a result, practitioners who are responsible for trauma patients on the coastal area should be made aware of the significantly increased risk for injuries associated with front seating. Proper spinal injury precautions must be universally complied to optimize treatment outcomes. Current safety regulations, in particular speed limitation, proper safety restraint techniques, bracing position on board and protocols for marine rescuers should be carefully reviewed. Optimistically number of victims and degree of injuries would be reduced.

\section{References}

1. Jecmenica DS, Alempijevic DjM, Aleksandric BV, Pavlekić SB, Baralić I, et al. (2010) Injuries of the cervical spine in motorcycling and bicycling traffic accidents. Acta Chir Iugosl 57(1): 135-140.

2. Hooten KG, Murad GJ (2014) Helmet use and cervical spine injury: a review of motorcycle, moped, and bicycle accidents at a level 1 trauma center. J Neurotrauma 31(15): 1329-1333.

3. Kaufman RP, Ching RP, Willis MM, Mack CD, Gross JA, et al. (2013) Burst fractures of the lumbar spine in frontal crashes. Accid Anal Prev 59: 153163.
4. Giustini M, Ade P, Taggi F, Funari E, et al. (2003) Accidents in recreational waters. Ann Ist Super Sanita 39(1): 69-76.

5. Burke DC (1972) Spinal cord injuries from water sports. Med J Aust 2(21): 1190-1194.

6. Hummel G, Gainor BJ (1982) Waterskiing-related injuries. Am J Sport Med 10(4): 215-218.

7. Stanisavljevic S, Irwin RB, Brown LR (1978) Orthopedic injuries in water-skiing: etiology and prevention. Orthopaedics 1(2): 125-129.

8. Steinbruck K, Paeslack V (1980) Analysis of 139 spinal cord injuries due to accidents in water sports. Paraplegia 18(2): 86-93.

9. Wilks J, Coory M (2000) Overseas visitors admitted to Queensland hospitals for water-related injuries. Med J Austr 173(5): 244-246.

10. MK Allami, EG Drakoulakis, H Dinopoulos, R Dunsmuir, DA Macdonald, et al. (2005) Thoracolumbar spinal injuries following speedboat accidents is it time to change the regulations? Injury Extra 36: 511-516.

11. Chalmers D, Morrison L (2003) Epidemiology of non-submersion injuries in aquatic sporting and recreational activities. Sports Med 33(10): 745-770.

12. Giustini M, Ade P, Taggi F, Funari E (2003) Accidents in recreational waters. Ann Ist Super Sanita 39(1): 69-76.

13. Selvarajah S, Schneider EB, Becker D, Sadowsky CL, Haider AH, et al. (2014) The epidemiology of childhood and adolescent traumatic spinal cord injury in the United States: 2007-2010. J Neurotrauma 31(18): 1548-1560.

14. Nijendijk JH, Post MW, van Asbeck FW (2014) Epidemiology of traumatic spinal cord injuries in the Netherlands in 2010. Spinal Cord 52(4): 258263.

15. Rahimi-Movaghar V, Sayyah MK, Akbari H, Khorramirouz R, Rasouli MR, et al. (2013) Epidemiology of traumatic spinal cord injury in developing countries: a systematic review. Neuroepidemiology 41(2): 65-85.

16. Wang H, Xiang Q Li C, Zhou Y (2013) Epidemiology of traumatic cervical spinal fractures and risk factors for traumatic cervical spinal cord injury in China. J Spinal Disord Tech 26(8): 306-313.

17. Sekhon LH, Fehlings MG (2001) Epidemiology, demographics, and pathophysiology of acute spinal cord injury. Spine (Phila Pa 1976) 26(Supply 24): 2-12.

18. Tator CH, Edmonds VE (1979) Acute spinal cord injury: analysis of epidemiologic factors. Can J Surg 22(6): 575-578.

19. Yang R, Guo L, Wang P, Lin Huang, Yong Tang, et al. (2014) Epidemiology of spinal cord injuries and risk factors for complete injuries in Guangdong, China: a retrospective study. Plos One 1: e84733.

20. Ivancic PC (2014) Cervical spine instability following axial compression injury: a biomechanical study. Orthop Traumatol Surg Re 100(1): 127133.

21. Maier R, Liederer A, Schabus R (1994) Treatment of spinal injuries. Wien Med Wochenschr 144(24): 619-630.

22. McAfee PC, Yuan HA, Fredrickson BE, Lubicky JP (1983) The value of computed tomography in thoracolumbar fractures. J Bone Joint Surg Am 65(4): 461-473.

23. Nicoll EA (1949) Fractures of the dorso-lumbar spine. J Bone Joint Journal 31B (3): 376-394.

24. Jazayeri SB, Beygi S, Shokraneh F, Hagen EM, Rahimi-Movaghar V, et al. (2015) Incidence of traumatic spinal cord injury worldwide: a systemic review. Eur Spine J 24(5): 905-918.

25. Jefferson G (1927) Discussion on spinal injuries. Proc R Soc Med 8: 625648.

26. HMSO (2000) Road Accidents in Great Britain: The Casualty Report, London: HMSO Publications. 
27. Kuppferschmid JP, Weaver ML, Raves JJ, Diamond Daniel L (1989) Thoracic spine injuries in victims of motorcycle accidents. J Trauma 29(5): 593-596.

28. Meyer S (1992) Thoracic spine trauma. Sem Roentgenol 25(4): 254-261.

29. Robertson A, Angus, Giannoudis PV, Branfoot T, Toby, et al. (2002) Spinal injuries in motorcycle crashes: patterns and outcome. J Trauma 53(1): 5-8.

30. Wieclawek H, Kiwerski J (1984) Thoracic spine injury after a plunge into water. Chir Narzadow Ruchu Ortop Pol 49(2): 111-113.

31. Denis F (1984) Spinal instability as define by the three-column spine concept in acute spinal trauma. Clin Orthop 189: 65-76.

32. Denis $F$ (1983) The three-column spine and its significance in the classification of acute thoracolumbar spinal injuries. Spine 8(8): 817831.

\section{ISSN: 2574-1241}

DOI: $10.26717 / B J S T R .2018 .12 .002217$

Narote Treenarong. Biomed J Sci \& Tech Res

(c) (i) This work is licensed under Creative

Submission Link: https://biomedres.us/submit-manuscript.php
33. Muller ME, Allgower M, Schneider R, Willenegger H, Allgöwer (1991) Manual of internal fixation recommended by the AO-ASIF group, $\left(3^{\text {rd }}\right.$ Edn.). Springer-Verag: Berlin.

34. Daffner RH (1988) Imaging of vertebral trauma. Aspen pp. 157-161.

35. Drysdale WF, Kraus JF, Frant CE, Riggins RS (1975) Injury patterns in motorcycle collisions. J Trauma 15(2): 99-115.

36. Shrosbree RD (1978) Spinal cord injuries as result of motorcycle accidents. Paraplegia 16(1): 102-112.

37. Robertson A, Branfoot T, Barlow IF, Giannoudis PV (2002) Spinal injuries resulting from car and motorcycle accidents. Spine 27(24): 2825-2830.

38. Ferguson RL, AllenBL Jr (1984) A mechanistic classification of thoracolumbar spine fractures. clin Orthop Relat Res 189: 77-88.

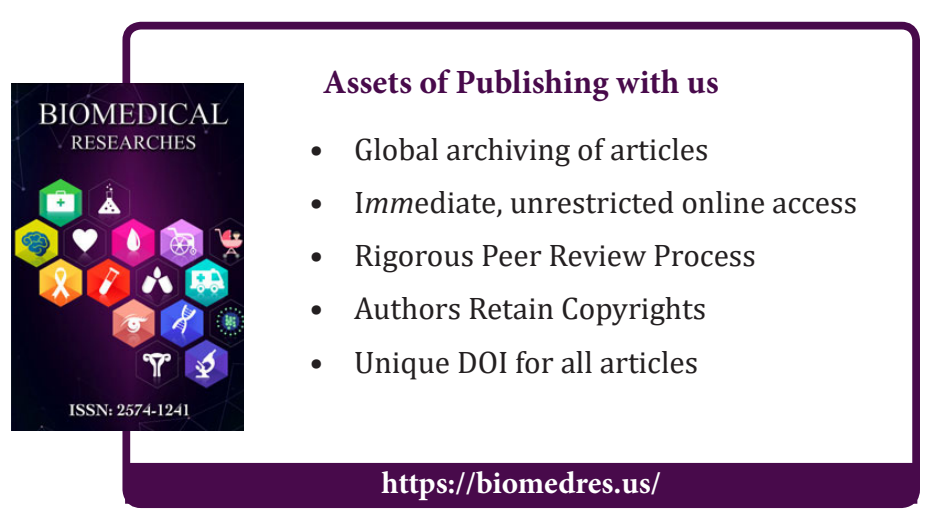

